\title{
General population knowledge about osteoarthritis and its related risk factors in Jeddah Saudi Arabia
}

Ali H. Alyami, MBBS, SSC (Orth), Muath M. Alswat, MBBS, Ibrahim A. Omer, Medical Students, Mohamed E.H. Ahmed, MSc, PhD, Salem H. Alshammari, Medical Students, Khalid W. Alsaggaf, MBBS, Jameel H. Amoudi, MBBS, Danya A. Aljafari, Medical Students.

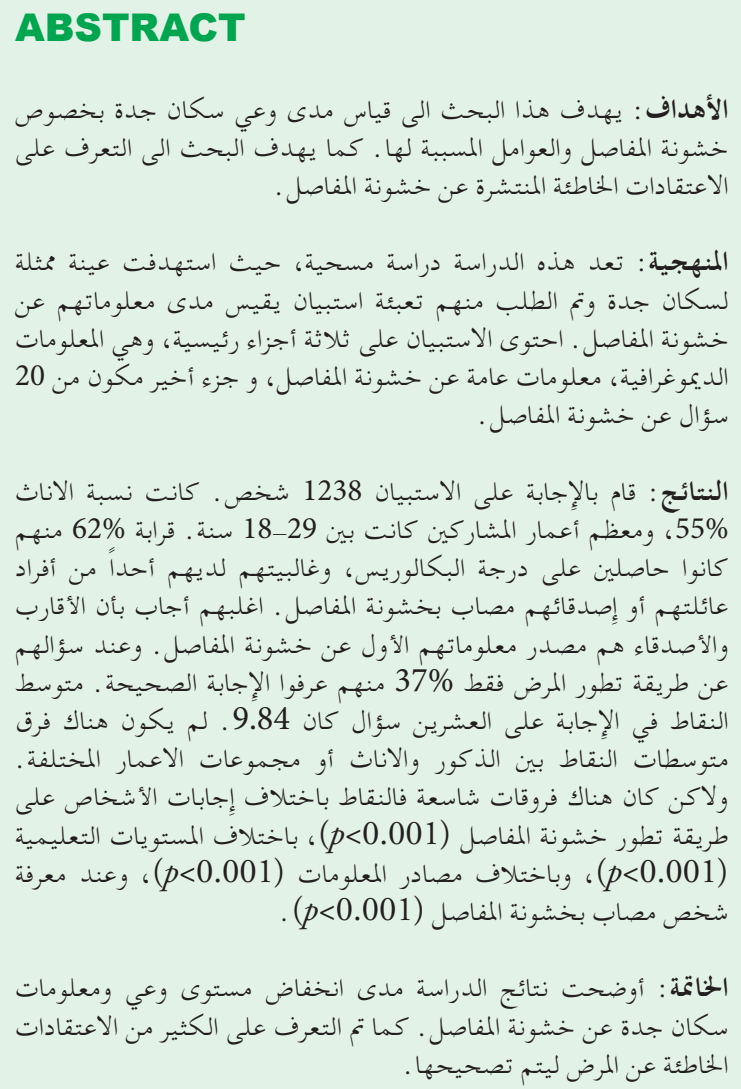

Objectives: To identify the knowledge gap and misconceptions about osteoarthritis (OA) and its risk factors among the general population of Jeddah, Saudi Arabia.

Methods: This is a cross-sectional study conducted Jeddah, Saudi Arabia, between 11-13 April, 2019. The study was approved by King Abdullah International Medical Research Center. A representative sample of the public in well-known mall was asked to complete the questionnaire to assess their knowledge regarding OA. The questionnaire consisted of 3 parts: 1) demographic data, 2) general knowledge regarding $\mathrm{OA}$, and 3) a quiz of 20 questions. A descriptive analysis was carried out with t-test and F-test-based method.

Results: The study had 1238 respondents. Approximately $55 \%$ of them were females, with $51.8 \%$ between the ages of 18 to 29 . Approximately $62 \%$ held a bachelor's degree and 63\% had a family member or a friend afflicted with OA. Relatives and friends were the main source of information regarding OA. Only 37\% correctly identified the mechanism behind OA. The mean score for the 20-item quiz was 9.84. No significant differences were found when comparing scores of males and females or those of age groups. However, significant differences in scores were identified between respondents of varying educational levels $(p<0.001)$, participants' knowledge of the mechanism leading to OA $(p<0.001)$, information sources $(p<0.001)$, and knowing someone with it $(p<0.001)$.

Conclusion: The results of our study suggest a low level of knowledge regarding $\mathrm{OA}$ among Jeddah population. Many misconceptions regarding OA were identified, so more information be brought to the public.

Keywords: osteoarthritis, population knowledge, questionnaire, knowledge, Saudi Arabia.

Saudi Med J 2020; Vol. 41 (5): 516-523 doi: 10.15537/smj.2020.5.25061

From the Orthopedic Division, Department of Surgery (Alyami), Ministry of the National Guard-Health Affairs; from King Abdullah International Medical Research Center (Alyami, Alswat, Omer, Ahmed, Alshammari, Alsaggaf, Amoudi, Aljafari); and from King Saud bin Abdulaziz University for Health Sciences (Alyami, Alswat, Omer, Ahmed, Alshammari, Alsaggaf, Amoudi, Aljafari), Jeddah, Kingdom of Saudi Arabia.

Received 7th January 2020. Accepted 31st March 2020.

Address correspondence and reprint request to: Dr. Muath M. Alswat, College of Medicine, King Saud bin Abdulaziz University for Health Sciences, Jeddah, Kingdom of Saudi Arabia. E-mail:alswat016@ksau-hs.edu.sa

ORCID: https://orcid.org/0000-0002-6725-9266 
Steoarthritis (OA) is the most prevalent form of arthritis, characterized by progressive degeneration, involving not only the articular cartilage, but the entire joint, including the subchondral bone and synovium. ${ }^{1,2}$ Several pathological changes are observed in OA-affected joints, including degradation of the articular cartilage, subchondral bone thickening, osteophyte formation, variable degrees of synovium inflammation, ligament degeneration, including the knee menisci, and hypertrophy of the knee capsule. ${ }^{2}$ These comprise the leading cause of joint pain, functional impairment, decreased mobility, increased risk of falls, and decreased physical activities. ${ }^{3}$ Several risk factors are linked to OA, the most prevalent was age, gender, obesity, prior joint injury, genetic predisposition, misalignment, and abnormal joint shape. ${ }^{1,2}$ Gender influences development of $\mathrm{OA}$, with females having a higher risk than males. Once diagnosed, females also experience a higher severity of the disease. ${ }^{4}$ Known to be a genetically complex condition, genetic factors play a major role in its development. ${ }^{5} \mathrm{~A}$ study reported that obesity and previous knee injury significantly contribute to OA onset, wherein is obesity one of the major risk factors. ${ }^{2}$

In Kingdom of Saudi Arabia (KSA), OA related epidemiological data are considered outdated, limiting researchers' understanding of the prevalence, morbidity, and the public's knowledge regarding the disease. Moradi-Lakeh et $\mathrm{al}^{6}$ stated that a high prevalence of knee OA have been reported in KSA.

A few studies reported the prevalence of certain types of OA in KSA; however, there is no literature related to the knowledge of the general public of Jeddah city regarding $\mathrm{OA}$ and the associated risk factors. In a study conducted in Aseer region, participants had a satisfactory level of knowledge regarding OA. ${ }^{7}$ Literature recommended a study to be conducted to measure the knowledge related to the disease in the region. This cross-sectional study aims to assess and analyze the knowledge of the general population regarding OA and the associated risk factors in Jeddah, KSA.

Methods. The study used an observational crosssectional design, and was conducted in Jeddah, KSA. The setting was a public, well-known mall in Jeddah (Mall of Arabia) with a good representative pool of the

Disclosure. Authors have no conflict of interests, and the work was not supported or funded by any drug company. general population. This mall was chosen because it is the biggest mall in Jeddah and its geographic location is accessible for people from all around Jeddah. Data collection started on 11 April, 2019, and was completed within 3 days. The study included people in Jeddah, from different nationalities, genders, and older than 18 years. Participants were selected randomly. We excluded individuals with a diagnosis of $\mathrm{OA}$, since they would possibly know more about the disease. A nonprobability convenience sampling technique was used. The sample size was calculated with a confidence level of $99 \%$, along with an approximate population size (Jeddah has a population of 5,000,000), margin of error $5 \%$, with a calculated sample size of 664 participants. Participants included 1238 (91\%) out of 1362.

Data sources/measurements. After reviewing literature regarding the knowledge of the general public and searching all related articles in PubMed and Google scholar, some questions from a validated patient knowledge questionnaire related to OA (PKQ-OA) were adapted. ${ }^{8}$ A self-administered questionnaire consisting of 3 sections in Arabic language was designed. The first section included demographic data, such as age, gender, and level of education. Participants were divided into 5 age groups (group 1: 18-29, group 2: 30-39, group 3: 40-49, group 4: 50-59, and group 5: 60 and older). The level of education was also categorized into 5 groups (no education, less than secondary, secondary, bachelor's, or higher education). In the second section, participants were asked about the definition of OA, and whether they understood it. In the third section, participants' knowledge was assessed using a 20 -item quiz, focusing on general facts, symptoms, risk factors, diagnosis, and OA treatment options. The response options were yes, no, or I don't know, to avoid guessing. Each correct response scored 1 point, with 0 for wrong, or 'I don't know' answers, with a total score of 20 points. All questions had one correct answer based on the currently accepted orthopedic literature. To ensure content validity, 3 orthopedic surgeons were asked to revise the questionnaire to determine if the topic was covered adequately, and if there were any irrelevant questions. Construct or criteria validity was not determined due to a lack of a standardized comparison. A pilot study was carried out, with 25 participants from the general public completing the questionnaire and commenting on its length, design, wording, understanding, and ease of completion. The data in the pilot sample was not included in the study sample. During data collection, an online form of the questionnaire was used in the mall. The data collectors used a quick response $(\mathrm{QR})$ 
code, scanned by participants' smart phones, which was completed in the presence of a data collector to ensure completion. Before inclusion, participants were assessed about fulfilling inclusion and exclusion criteria, and were excluded if ineligible.

Participants were asked if they were willing to participate and the completion of the questionnaire was accepted as consent. No data that could possibly identify a participant, such as a name or phone numbers was collected. The study was approved by an Institutional Review Board (IRB).

Statistical analysis. The raw data was processed and cleaned prior to the statistical analysis. Data management and analyses were carried out by using the IBM Statistical Software for Social Sciences (SPSS) for Windows, version 25 (IBM Corp., Armonk, N.Y., USA).Qualitative variables are presented as frequency and percentage and the quantitative variables as mean and standard deviation. The data comparison was carried out with a student-t-test and F-test within a $p$-value of 0.05 .

Results. The sample size was 1,238 . The majority of participants $(55 \%)$ were female. In terms of age, the majority (52\%) were 18 to 29 years-old, with $24 \%$ in the 30 to 39 years-old, and $17.5 \%$ in the 40 to 49 years-old. Only a small proportion $(1 \%)$ was 60 years or older. The most frequent levels of education were bachelor's degree (62\%) and a completed high school education (24\%). A small proportion (7.7\%) achieved an educational level higher than a bachelor's degree. The majority of the sample (63\%) had a family member or friend who was diagnosed with OA (Table 1).

Approximately $37 \%$ was knowledgeable about underlying mechanisms of OA, and correctly identified it as a process in which the protective cartilage at the bone ends wore down over time. A small proportion $(15.3 \%)$ indicated acid accumulation in the joint and $11.1 \%$ chose compression of a nerve passing near the joint. It is noteworthy that $26 \%$ had no knowledge about $\mathrm{OA}$ at all. In terms of source of information, almost half (44.8\%) indicated relatives and friends. A small proportion (19\%) indicated the Internet and social media, with only $7.6 \%$ acquiring information from a physician (Table 2).

The mean score for the 20-item quiz was 9.84 \pm 3.27 . The first part of the quiz consisted of 5 general facts regarding OA. In terms of it being a chronic problem, $67.5 \%$ responded yes and $60 \%$ did not think OA was rare. Only $36.6 \%$ knew that OA can affect different joints, $35.2 \%$ negatively responded to OA being caused by cold, damp weather, and only $34.8 \%$ knew that OA was not caused by a microorganism.

The second part of the quiz included 4 questions about the main symptoms of OA. More than half (51\%) knew that pain was not the only symptom, $51.3 \%$ also indicated stiffness, and $40.1 \%$ knew that swelling was a sign. The majority $(66.8 \%)$ agreed that OA could lead to loss of joint movement. The third part assessed knowledge about the main risk factors of OA. More than a third (37.3\%) knew that genetic factors can predispose to $\mathrm{OA}$, while the majority ( $80 \%) \mathrm{knew}$ aging to be a risk factor. In terms of gender being equally affected by OA, less than $50 \%$ indicated whether this was the case. The last part of the quiz focused on the diagnosis and treatment of OA. Approximately $46.7 \%$ used physical examination and $\mathrm{x}$-ray and $30.5 \%$ through blood tests. Regarding treatment, $46 \%$ chose nonsteroidal antiinflammatory drugs and $52.6 \%$ thought acid-free diets were a proven treatment. The majority (69\%) found that exercise, such as swimming, was beneficial, such that $75 \%$ responded that physiotherapy could improve OA symptoms. A third (34.5\%) were sceptical about an intra-articular stem cell injection or hyaluronic acid used as an effective modality for OA, while $34.5 \%$ knew

Table 1 - Mean knowledge score by demographic variables.

\begin{tabular}{|c|c|c|c|c|}
\hline Item & $\mathbf{n}$ & $\%)$ & $\begin{array}{c}\text { Mean } \\
\text { knowledge } \\
\text { score }\end{array}$ & $P$-value \\
\hline \multicolumn{5}{|l|}{ Gender } \\
\hline Male & 558 & $(45.1)$ & 9.67 & 0.099 \\
\hline Female & 680 & $(54.9)$ & 9.98 & \\
\hline \multicolumn{5}{|l|}{ Age } \\
\hline $18-29$ & 641 & $(51.8)$ & 9.73 & 0.702 \\
\hline $30-39$ & 300 & $(24.2)$ & 9.86 & \\
\hline $40-49$ & 215 & $(17.4)$ & 10.08 & \\
\hline $50-59$ & 69 & (5.6) & 9.99 & \\
\hline$\geq 60$ & 13 & $(1.0)$ & 10.15 & \\
\hline \multicolumn{5}{|l|}{ Education } \\
\hline Uneducated & 9 & $(0.7)$ & 6.89 & $0.000^{*}$ \\
\hline Less than secondary & 41 & $(3.3)$ & 9.22 & \\
\hline Secondary & 298 & $(24.1)$ & 9.09 & \\
\hline Bachelor & 795 & $(64.2)$ & 10.17 & \\
\hline Higher education & 95 & $(7.7)$ & 9.97 & \\
\hline \multicolumn{5}{|c|}{${ }^{*}$ significant at $1 \%$} \\
\hline
\end{tabular}


Osteoarthritis knowledge among public ... Alyami et al

Table 2 - Knowledge by knowledge related responses and source of information.

\begin{tabular}{lrrc}
\hline Item & $\mathbf{n}$ & $(\%)$ & $\begin{array}{c}\text { Mean } \\
\text { knowledge score }\end{array}$ \\
\hline Underlying mechanism & & & \\
$\quad$ Blood that reach the joint decreases with aging & 132 & $(10.7)$ & 9.49 \\
Nerve passing near the join gets compressed & 137 & $(11.1)$ & 10.3 \\
Cartilage on the ends of the bones wears down over time & 458 & $(37.0)$ & 10.67 \\
Acid accumulation inside the joint & 189 & $(15.3)$ & 10.14 \\
I don't know & 322 & $(26.0)$ & 8.43 \\
Source of information & & & \\
Personal experience & 121 & $(9.8)$ & 10.05 \\
Relatives and friends & 555 & $(44.8)$ & 10.12 \\
Television & 58 & $(4.7)$ & 8.74 \\
Internet and social media & 238 & $(19.2)$ & 9.39 \\
School or university & 132 & $(10.7)$ & 10.38 \\
Books, magazines and newspapers & 40 & $(3.2)$ & 8.9 \\
Physician & 94 & $(7.6)$ & 9.34 \\
Knowing patient with osteoarthritis & & & \\
Yes & 780 & $(63.0)$ & 10.27 \\
No & 458 & $(37.0)$ & 9.1 \\
\hline
\end{tabular}

Table 3 - Participants' answers to 20-items quiz.

\begin{tabular}{|c|c|c|c|c|}
\hline \multicolumn{2}{|c|}{ Items on the quiz } & \multirow{2}{*}{$\begin{array}{l}\text { Yes } \\
67.5\end{array}$} & \multirow{2}{*}{$\begin{array}{l}\text { No } \\
18.4\end{array}$} & \multirow{2}{*}{$\begin{array}{r}\text { Not sure } \\
14.1\end{array}$} \\
\hline 1 & Do you think osteoarthritis is a chronic problem? & & & \\
\hline 2 & Do you think osteoarthritis is rare? & 19.1 & 60.1 & 20.8 \\
\hline 3 & Do you think that different joints can be affected by osteoarthritis? & 36.6 & 41.4 & 20.0 \\
\hline 4 & Do you think osteoarthritis is caused by cold, damp weather? & 41.4 & 35.2 & 23.4 \\
\hline 5 & Do you think it is developed by a microorganism? & 28.1 & 38.5 & 33.4 \\
\hline 6 & Do you think pain is the only symptom of osteoarthritis? & 33.2 & 51.7 & 15.1 \\
\hline 7 & Do you think stiffness is a symptom of osteoarthritis? & 51.3 & 32.8 & 24.9 \\
\hline 8 & Do you think swelling is a sign of osteoarthritis? & 40.1 & 35.5 & 24.4 \\
\hline 9 & Do you think osteoarthritis can lead to loss of joint movement? & 66.8 & 18.2 & 15.0 \\
\hline 10 & Do you think there are genetic factors that can predispose parson to osteoarthritis? & 37.3 & 40.6 & 22.1 \\
\hline 11 & Do you think aging is a risk factor for osteoarthritis? & 81.0 & 10.3 & 8.7 \\
\hline 12 & Do you think men and women are equally affected by osteoarthritis? & 29.5 & 49.0 & 21.5 \\
\hline 13 & Do you think physical examination and x-ray are used to diagnose osteoarthritis? & 46.7 & 32.0 & 21.3 \\
\hline 14 & Do you think blood tests are used to diagnose osteoarthritis? & 30.5 & 41.5 & 28.0 \\
\hline 15 & Do you think NSAIDs can improve osteoarthritis symptoms? & 46.0 & 36.5 & 17.5 \\
\hline 16 & Do you think some forms of exercise like swimming is suitable for people with osteoarthritis? & 69.0 & 13.9 & 17.1 \\
\hline 17 & Do you think acid-free diets are a proven treatment for osteoarthritis? & 52.6 & 18.2 & 29.2 \\
\hline 18 & Do you think physiotherapy can cause a great improvement in the symptoms of osteoarthritis? & 75.7 & 10.0 & 14.3 \\
\hline 19 & Do you think intra-articular injection by stem cell or hyaluronic acid is an effective modality for curing OA? & 27.6 & 35.1 & 37.3 \\
\hline 20 & Do you think a joint replacement surgery will be the ultimate option to relieve the symptoms of osteoarthritis? & 345.0 & 33.3 & 32.3 \\
\hline
\end{tabular}

OA - osteoarthritis, NSAIDs - nonsteroidal anti-inflammatory drugs 
that joint replacement surgery was the final option to relieve OA symptoms (Table 3).

When comparing the mean score based on gender, the male subgroup scored $9.67 \pm 3.3$ and the female subgroup scored $9.98 \pm 3.2$, which was not statistically significant $(p=0.099)$. Although the 40-49-year-old subgroup scored higher $(10.1 \pm 3.3)$ than the other age groups, the difference was not statistically significant $(p=0.702)$. However, a significant difference in the educational level score means was found $(p<0.001)$ : the bachelor educational level subgroup had the highest mean score $(10.17 \pm 3.1)$, compared to the uneducated subgroup $(6.89 \pm 2.6)$. In terms of responses related to the underlying mechanism of $\mathrm{OA}$, the group that correctly knew the underlying mechanism scored $10.67 \pm 3.1$, which was significantly higher than the other groups $(p<0.001)$. The subgroup that indicated their source of information about OA was school and university, scored $10.38 \pm 3.4$, which was significantly higher than the other groups $(p<0.001)$. In addition, the subgroup with a family member or friend diagnosed with OA, had a significantly higher mean score (10.27 $\pm 3.2, p<0.001)$.

Discussion. Due to very little literature about OA, the current study suggests further studies be carried out to help inform the general public of KSA. This study included a large sample in terms of age, gender, and educational level that is representative of the general public. This study explored the knowledge related to $\mathrm{OA}$ and the associated risk factors of a segment of the general public in Saudi Arabia. ${ }^{7}$ More than half of the sample (55\%) was female, the majority (62\%) held a bachelor's degree, and 52\% were in the 18 to 29-year-age group, reflecting the large proportion of young adolescents $(52.7 \%)$ in the societal demographics of the Saudi population. ${ }^{9}$ Given the high prevalence of OA in Saudi Arabia, $15.3 \%$ in all age groups, it was not surprising that the majority $(66 \%)$ of the sample knew a relative or a friend diagnosed with OA. ${ }^{6}$

The sample had a low level of knowledge regarding the underlying mechanism of OA with only $37 \%$ correctly identifying the cause. The findings also indicated that a small proportion (15.3\%) were confused and could not differentiate between OA and gout. This common misconception could be due to the association of both conditions with advancing age and obesity and frequently co-exist in an individual; however, the pathogenic relationship between OA and gout is still unknown. ${ }^{10}$ Surprisingly, just less than half $(44.8 \%)$ reported their source of information about $\mathrm{OA}$ to be their friends and family, which was contrary to similar studies assessing the knowledge of the general public. ${ }^{11-13}$ The finding is a concern because the friends and family may not be knowledgeable to provide the correct medical information. In addition, few participants (19\%) relied on the Internet and social media as their source of information. However, it has been shown that the quality of the information available on the Internet is a concern as many negative consequences have been reported. ${ }^{14-16}$ It should be noted that only $7.6 \%$ obtained their OA related information from a physician, highlighting the need for physicians to intensify their efforts to educate their patients and their families and to correct their misconceptions.

The majority of the sample $(67.5 \%)$ knew that OA is a chronic disease with $60 \%$ knowing that it is not a rare disease. Osteoarthritis is a prevalent disease and the single most frequent cause of disability in older adults. ${ }^{17}$ Osteoarthritis affects weight bearing joints such as the lumbosacral spine, hip and knee; however, it can affect other joints such as the interphalangeal, carpometacarpal and temporo mandibular. ${ }^{18}$ Only a third in the current study (36.6\%) knew that OA may also affect other joints and $42 \%$ thought $\mathrm{OA}$ is caused by cold, damp weather, a misconception attributable to the fact that weather instability may have an impact on the joint pain perception of people with OA; however, the weather does not cause OA. ${ }^{19}$ It is also concerning that only $34.8 \%$ knew that $\mathrm{OA}$ is not caused by a microorganism and $34.8 \%$.

Symptoms of osteoarthritis. The predominant symptom of OA is pain which is usually the reason for a medical consultation. Other symptoms include stiffness, particularly in the morning or after a period of inactivity and resolves within 20 to 30 minutes. ${ }^{20}$ About half of our sample knew that pain is not the only symptom of OA and just more than half (51.3\%) identified stiffness as an OA symptom. Swelling of the joint $(40 \%)$ often presents in the beginning of the disease and patients with OA have a restricted range of motion $(67 \%)$ which is directly associated with their disability. ${ }^{21}$

Risk factors of osteoarthritis. The majority (81\%) knew that aging is a risk factor. In addition, females have a higher change of developing $\mathrm{OA}$ and are afflicted more severely by the disease. ${ }^{1}$ Only half of the participants knew about the gender differences in the context of OA. A strong genetic basis for OA is a known risk factor and heredity accounts for about half of the OA cases affecting the hands and the hips and a smaller percentage, the knees. ${ }^{1}$ Only a third of the sample $(37.3 \%)$ knew about the genetic association with OA. 
Diagnosis and treatment of osteoarthritis. Plain film radiography, history and physical examination are sufficient to diagnose OA. The use of blood biochemical markers in the diagnosis of $\mathrm{OA}$ is limited. Many markers have been proposed like C-terminal telo peptide of collagen type II and cartilage oligomeric matrix protein, markers of tissue degradation, but they have not yet been sufficiently validated for use in clinical practice. ${ }^{22}$ Less than half of the sample knew that an x-ray and physical examination are used to diagnose OA and only $42 \%$ knew that a blood test is not used to diagnose OA. Regarding the management of OA, NSAIDs play an important role in controlling the symptoms which was known by $46 \%$ of the participants. ${ }^{22}$ Exercise in the form of swimming is suitable for patients with $\mathrm{OA}$ and is associated with an improvement in symptoms, joint stiffness and muscle strength, ${ }^{23}$ known by the majority (69\%) of the sample. Another erroneous belief of half of the sample was that acid-free diets could treat $\mathrm{OA}$ which can be attributed to their lack of understanding of the underlying mechanism of OA. Physiotherapy is considered one option in the treatment of $\mathrm{OA}$, as it improves pain and joint function in 50\%-75\% of patients. ${ }^{24}$ In the current study, $75 \%$ knew that physiotherapy could significantly improve OA. Less than a third $(28 \%)$ responded that an intra-articular injection of stem cell or hyaluronic acid is used as a modality for curing OA. The use of stem cell injection has drawn considerable attention in the past few years. Many studies are being conducted to investigate the safety and efficacy; however, the majority are still in an early phase and it is premature to draw a conclusion about its potential curative effect. ${ }^{25-27}$ However, intra-articular hyaluronic acid therapy has been shown to cause a significant improvement in pain, function, and stiffness. ${ }^{28}$ Though there was no increase in adverse events, the safety profile of hyaluronic acid therapy requires more evidence. ${ }^{29}$ Total and partial joint replacement for advanced disease remain the mainstay of treatment. ${ }^{22}$ In this study, $35 \%$ of the participants knew that joint replacement surgery is the final option to relieve the symptoms of OA.

Participant scores stratified by gender, age, level of education, underlying mechanism, source of information and knowing someone with osteoarthritis. The scores of the participants were calculated and analyzed with the total score of 20. The overall mean knowledge score was 9.84. Both age and gender were not statistically significant but a statistically significant difference was found for educational level. The bachelor subgroup had a significant difference in the total mean score compared to the other educational subgroups. This might be due to the comprehensive curricula offered in Saudi public colleges where undergraduate students are encouraged to enrol in elective courses not directly related to their majors, resulting in being exposed to various disciplines. Overall, this conventional conception, where the awareness level directly dictates the proportionality of acquired awareness, appears to be the norm across various health disciplines as evidenced by literature related to levels of knowledge and/or awareness. ${ }^{2,30}$

The subgroup who correctly identified the underlying mechanism, scored higher relative to the other subgroups, which may indicate prior knowledge about OA. In addition, participants who indicated their primary source of information as an educational institution scored significantly higher than the other subgroups. This maybe due to the quality of educational material offered in such settings in contrast to information broad casted via television commercials and programs as the television and social media subgroup had the lowest score. The findings of the current study highlight the urgent need for high-spectrum educational campaigns to be main streamed through several outlets, including television, social media, and paper-based news outlets. Knowing a patient diagnosed with also significantly contributed to better overall knowledge about the disease. This may be due to information communicated by the patient, accompanying the patient to their physician visits, or self-learning prompted by the strong societal dynamics between individuals in Saudi Arabia. In previous similar study that was carried out in Aseer region and included 1052 participants, the level of knowledge about OA was good particularly regarding preventive measures of OA. ${ }^{7}$ Similar to this study, they found participants who held a bachelor's degree to have better knowledge about the disease. Whereas they found participants whose age was 50 year or older had higher level of knowledge and awareness about OA; however, our study demonstrated no differences in the knowledge between participants of varying age groups. ${ }^{7}$ Another study assessed level of knowledge of OA among railway workers in Malaysia reported the majority of the participant to have low knowledge level. ${ }^{31}$ They noticed having a family member with OA is a good predictive factor of having better knowledge. ${ }^{31}$

The findings of current study demonstrated the lack of knowledge about OA and the associated risk factors in the general public. It would have been interesting to compare the current study to previous studies; however, our study was the first of its kind. Awareness campaigns are one of the methods to increase the general public's knowledge about OA. Communication with health care professionals and initiating educational programs 
can bridge this gap and facilitate a better understanding of such a prevalent disease in the general public. Osteoarthritis would be understood if information was provided in a clear, non-medical language and made available to the public free of charge. In addition, developing free tools, info-graphics, and resources about $\mathrm{OA}$, injury prevention, obesity management, physical activities, and self-management education would be empowering and improve knowledge. The widespread use of social media makes it an ideal medium for awareness advertisements and advantage can be taken of social media outlets such as Instagram, Snap Chat, and Twitter to distribute initiatives directly to the population of Jeddah.

Study limitations. The results of this study are not generalizable since it was conducted in one geographic location and a convenience sampling technique was used for data collection. Another limitation is the socioeconomic variability of the Jeddah public as the reliability of some measures may be influenced by a lower socioeconomic status and no data was collected related to the participants' income.

In conclusion, the general public of Jeddah exhibited low levels of knowledge about OA and its associated risk factors: it is a preventable disease that affects quality of life for patients. Many misconceptions about the causes, risk factors, and management of OA were identified, although improving the public's knowledge would add significant value. Awareness campaigns could also be used to increase the population's knowledge, which may result in early detection of the disease, correcting misconceptions and preventing harmful and unproven practices in OA management. More studies are required to investigate the population's knowledge as well as to provide physicians with evidence of common misconceptions.

Acknowledgment. The authors would like to acknowledge Dr. Mohammad B. Abrar, Dr. Abdulaziz Basaqr, and Dr. Mohammad Khan for their appreciated efforts in reviewing the design and content of the questionnaire. We thank Dr. Susanna Wright from the publication office in KAIMRC for her review and editing of the manuscript. We also thank the volunteer data collectors without whom the study would not have been carried out. We thank all the participants for their effort and time in completing the questionnaire. We would like to thank American manuscript editors (https://americanmanuscripteditors.com/lindex.aspx) for English language editing.

\section{References}

1. Vina ER, Kwoh CK. Epidemiology of osteoarthritis: literature update. Curr Opin Rheumatol 2018; 30: 160-167.
2. Silverwood V, Blagojevic-Bucknall M, Jinks C, Jordan JL, Protheroe J, Jordan KP. Current evidence on risk factors for knee osteoarthritis in older adults: a systematic review and meta-analysis. Osteoarthritis and Cartilage 2015; 23: 507-515.

3. Scuccato R. [Falls in the elderly.]. Recenti Prog Med. 2018; 109 : 401-404.

4. Vina ER, Ran D, Ashbeck EL, Ratzlaff C, Kwoh CK. Race, sex, and risk factors in radiographic worsening of knee osteoarthritis. Semin Arthritis Rheum 2018; 47: 464-471.

5. Palazzo C, Nguyen C, Lefevre-Colau MM, Rannou F, Poiraudeau S. Risk factors and burden of osteoarthritis. Ann Phys Rehabil Med 2016; 59: 134-138.

6. Moradi-Lakeh M, Forouzanfar MH, Vollset SE, El Bcheraoui C, Daoud F, Afshin A, et al. Burden of musculoskeletal disorders in the Eastern Mediterranean Region, 1990-2013: findings from the Global Burden of Disease Study 2013. Ann Rheum Dis. 2017; 76: 1365-1373.

7. Mukharrib MS, Al-Sharif MN, Alshehri TK, Shaker A. Knowledge of knee osteoarthritis among general population in Aseer region. J Family Med Prim Care 2018; 7: 1385-1389.

8. Hill J, Bird H. Patient knowledge and misconceptions of osteoarthritis assessed by a validated self-completed knowledge questionnaire (PKQ-OA). Rheumatology (Oxford) 2007; 46: 796-800.

9. General Authority for Statistics. The statistical yearbook. Riyadh (KSA): General Authority for Statistics; 2018. p. 98-120.

10. Yokose C, Chen M, Berhanu A, Pillinger MH, Krasnokutsky S. Gout and Osteoarthritis: Associations, Pathophysiology, and Therapeutic Implications. Curr Rheumatol Rep 2016; 18: 65.

11. Algahtani H, Shirah B, Alhazmi A, Alshareef A, Bajunaid M, Samman A. Perception and attitude of the general population towards Alzheimer's disease in Jeddah, Saudi Arabia. Acta Neurol Belg 2020; 120: 313-320.

12. Mihai GI, Ancuta RV, Monica T, Florina R, Zita F, Raquel G, et al. Population preferences for sources that offers information about dietary fibres health effects - an international crosssectional survey. J Pak Med Assoc. 2019; 69: 985-990.

13. Dalhaug EM, Haakstad LA. What the Health? Information sources and maternal lifestyle behaviors. Interact J Med Res. 2019; 8: e10355.

14. Fernandez-Luque L, Staccini P. All that glitters is not gold: Consumer health informatics and education in the era of social media and health apps. Findings from the yearbook 2016 section on consumer health informatics. Yearb Med Inform 2016; (1): 188-193.

15. Rozental A, Boettcher J, Andersson G, Schmidt B, Carlbring P. Negative effects of internet interventions: a qualitative content analysis of patients' experiences with treatments delivered online. Cogn Behav Ther 2015; 44: 223-236.

16. Seymour B, Getman R, Saraf A, Zhang LH, Kalenderian E. When advocacy obscures accuracy online: digital pandemics of public health misinformation through an antifluoride case study. Am J Public Health 2015; 105: 517-523.

17. Martel-Pelletier J, Barr AJ, Cicuttini FM, Conaghan PG, Cooper C, Goldring MB, et al. Osteoarthritis. Nat Rev Dis Primers. 2016; 2: 16072.

18. Allen KD, Golightly YM. State of the evidence. Curr Opin Rheumatol 2015; 27: 276-283.

19. Timmermans EJ, Schaap LA, Herbolsheimer F, Dennison EM, Maggi S, Pedersen NL, et al. EPOSA Research Group. The influence of weather conditions on joint pain in older people with osteoarthritis: Results from the European Project on osteoarthritis. J Rheumatol 2015; 42: 1885-1892. 
20. O’Neill TW, Felson DT. Mechanisms of osteoarthritis (OA) Pain. Curr Osteoporos Rep 2018; 16: 611-616.

21. Joshi SV, Chaudhari HR, Chaudhari NA. Effect of Education on Awareness, Knowledge, and willingness to be vaccinated in females of western India. J Cancer Educ 2020; 35: 61-68.

22. Glyn-Jones S, Palmer AJ, Agricola R, Price AJ, Vincent TL, Weinans H, et al. Osteoarthritis. Lancet 2015; 386: 376-387.

23. Alkatan M, Baker JR, Machin DR, Park W, Akkari AS, Pasha EP, et al. Improved Function and Reduced Pain after Swimming and Cycling Training in Patients with Osteoarthritis. J Rheumatol 2016; 43: 666-672.

24. MacKay C, Hawker GA, Jaglal SB. Qualitative study exploring the factors influencing physical therapy management of early knee osteoarthritis in Canada. BMJ Open 2018; 8: e023457.

25. Im GI. Perspective on intra-articular injection cell therapy for osteoarthritis treatment. Tissue Eng Regen Med 2019; 16: 357-363.

26. Jiang Y, Tuan RS. Origin and function of cartilage stem/ progenitor cells in osteoarthritis. Nat Rev Rheumatol 2015; 11 : 206-212.
27. Trounson A, McDonald C. Stem Cell Therapies in Clinical Trials: Progress and Challenges. Cell Stem Cell 2015; 17: 11-22.

28. Bhandari M, Bannuru RR, Babins EM, Martel-Pelletier J, Khan M, Raynauld JP, et al. Intra-articular hyaluronic acid in the treatment of knee osteoarthritis: a Canadian evidence-based perspective. Ther Adv Musculoskelet Dis 2017; 9: 231-246.

29. Honvo G, Reginster JY, Rannou F, Rygaert X, Geerinck A, Rabenda V, et al. Safety of Intra-articular Hyaluronic Acid Injections in Osteoarthritis: Outcomes of a Systematic Review and Meta-Analysis. Drugs Aging 2019; 36 (Suppl 1): 101-127.

30. Michl GL, Katz JN, Losina E. Risk and risk perception of knee osteoarthritis in the US: a population-based study. Osteoarthritis and Cartilage 2016; 24: 593-596.

31. Ganasegeran K, Menke JM, Challakere Ramaswamy VM, Abdul Manaf R, Alabsi AM, Al-Dubai SA. Level and determinants of knowledge of symptomatic knee osteoarthritis among railway workers in Malaysia. BioMed Res Int 2014; 2014: 370273. 